\title{
Body Muscle-to-Fat Ratio, Rather Than Fat-to-Muscle Ratio, Significantly Correlates With Measured Insulin Resistance in Patients With Type 2 Diabetes Mellitus
}

\author{
Noboru Kurinamia, b, e, Seigo Sugiyama ${ }^{\text {a, c, e }}$, Akira Yoshida ${ }^{\text {a }}$, Kunio Hieshima ${ }^{\text {a }}$, Fumio Miyamoto ${ }^{\text {, }}$ \\ Keizo Kajiwara ${ }^{a}$, Katsunori Jinnouch ${ }^{\mathrm{a}}$, Tomio Jinnouchi ${ }^{\mathrm{a}}$, Masatoshi Nomura ${ }^{\mathrm{b}}$, \\ Hideaki Jinnouchi ${ }^{\text {a, }}$, f
}

\begin{abstract}
Background: Insulin resistance (IR) assessment is important in treating type 2 diabetes mellitus (T2DM). We thus compared body muscle-to-fat ratio (BMFR) and fat-to-muscle ratio (FMR) values against $\mathrm{M} / \mathrm{I}$ values as clinical index of IR.

Methods: Subject included 118 untreated T2DM patients. Hyperinsulinemic-euglycemic clamp examination was performed to calculate the $\mathrm{M} / \mathrm{I}$ as index of IR. Body composition was measured by impedance analysis using InBody770.

Results: Simple linear regression analyses confirmed correlations between M/I and BMFR (B: $0.756(\mathrm{P}<0.01)$, coefficients of determination $\left(\mathrm{R}^{2}\right)$ : 0.572 , mean absolute error (MAE): 3.19 , and root mean squared error (RMSE): 4.14), and between M/I and FMR (B: -0.601 $(\mathrm{P}<0.01), \mathrm{R}^{2}: 0.362$, MAE: 3.97, and RMSE: 5.05). Against the M/I values, BMFR also showed better goodness-of-fit than did FMR. In comparing correlation coefficients, the BMFR absolute B value was significantly larger than that of FMR $(\mathrm{P}=0.027)$.
\end{abstract}

Conclusions: BMFR is more useful than FMR in quantifying IR in patients with T2DM because the correlation between BMFR and the insulin sensitivity index $\mathrm{M} / \mathrm{I}$ is significantly greater than that between FMR and M/I.

Keywords: Diabetes mellitus; Insulin resistance; Obesity; Body mus-

Manuscript submitted December 1, 2020, accepted July 7, 2021

Published online July 28, 2021

${ }^{a}$ Diabetes Care Center, Jinnouchi Hospital, Kumamoto, Japan

${ }^{b}$ Division of Endocrinology and Metabolism, Department of Internal Medicine, Kurume University School of Medicine, Kurume, Japan

'Division of Cardiovascular Medicine, Diabetes Care Center, Jinnouchi Hospital, Kumamoto, Japan

${ }^{d}$ Division of Preventive Cardiology, Department of Cardiovascular Medicine, Kumamoto University Hospital, Kumamoto, Japan

eNoboru Kurinami and Seigo Sugiyama contributed equally to the present study.

${ }^{\mathrm{f} C}$ Corresponding Author: Hideaki Jinnouchi, Jinnouchi Hospital, 6-2-3 Kuhonji, Chuo-ku, Kumamoto 862-0976, Japan. Email: hideaki@jinnouchi.or.jp

doi: https://doi.org/10.14740/jocmr4401 cle-to-fat ratio; Fat-to-muscle ratio; Hyperinsulinemic-euglycemic clamp

\section{Introduction}

Insulin resistance (IR) is a critical factor in managing type 2 diabetes mellitus (T2DM), and should be individually assessed in each patient with T2DM. Hyperinsulinemic-euglycemic clamp examination, the gold standard for assessing IR, provides us an clinical index of IR as glucose infusion rate ( $\mathrm{M}$ value) divided by steady-state serum insulin (I) value (M/I values). We previously reported a strong correlation between IR and body muscle-to-fat ratio (BMFR; muscle mass ( $\mathrm{kg}$ )/fat mass $(\mathrm{kg})$ ) [1,2]. Because skeletal muscle loss and body fat accumulation affect IR, the combination of muscle and fat is attracting wider attention as a clinical index. The fat-to-muscle ratio (FMR; fat mass (kg)/muscle mass $(\mathrm{kg})$ ), which is the reciprocal value of BMFR, is reportedly useful in diagnosing metabolic syndrome with IR in otherwise healthy people [3, 4]. In contrast, IR is known to improve after starting treatment for DM, including metformin [5], thiazolidine [6], and sodium-glucose cotransporter-2 (SGLT2) inhibitors [7]. We hypothesized that BMFR (rather than FMR) could more accurately indicate IR in patients with untreated T2DM. We thus compared BMFR and FMR values against M/I values.

\section{Materials and Methods}

\section{Subjects and protocol}

Patients with untreated T2DM who visited the Diabetes Care Center at Jinnouchi Hospital between June 2014 and August 2019 were enrolled. Those who had already been treated for diabetes, severe uncontrolled diabetes, diabetic ketoacidosis that needed immediate insulin treatment, or uncontrolled severe hypertension, and those who could not remain standing to have an elementary body composition tests, were excluded. The study was conducted in accordance with the Declaration of Helsinki. The study protocol was approved by the Human 
Ethics Review Committee of Jinnouchi Hospital (2020-2-1; UMIN protocol registration ID: UMIN000039857).

\section{Hyperinsulinemic-euglycemic clamp}

Insulin sensitivity was evaluated using a hyperinsulinemic-euglycemic clamp with an artificial pancreas (Nikkiso STG-55, Tokyo, Japan), as reported previously [1, 2]. Insulin was administered as an intravenous loading dose (starting from $4.77 \mathrm{mU} /$ $\mathrm{kg} / \mathrm{min}$ that was gradually decreased to $1.67 \mathrm{mU} / \mathrm{kg} / \mathrm{min}$; under these conditions, the estimated plasma insulin concentration was about $100 \mathrm{mU} / \mathrm{L}$ ) over $10 \mathrm{~min}$ followed by a continuous infusion at $1.5 \mathrm{mU} / \mathrm{kg} / \mathrm{min}$ for $120 \mathrm{~min}$. Plasma glucose concentrations were maintained at $5.5 \mathrm{mmol} / \mathrm{L}$ using a variable infusion of $10 \%$ glucose. Blood insulin concentration at steady state was measured when the hyperinsulinemic-euglycemic clamp examination was terminated (I value). Because of variations in the insulin clearance rate for each patient, the actual blood insulin concentrations during the hyperinsulinemic-euglycemic clamp test were different from the calculated insulin levels [8]. To correct for the effect of the variability in insulin concentrations among individual patients, we used the $\mathrm{M} / \mathrm{I}$ value as an index of insulin sensitivity, which was a value calculated by dividing the $M$ value by the steady-state serum insulin value (I) in this study. This value indicates glucose use per 1 unit of blood insulin and is a good index that represents tissue insulin sensitivity, which reflects whole-body insulin resistance [9].

\section{Measurement of body fat and muscle composition}

We measured body composition including body fat mass and body fat percentage using the same method as we described previously [10-13]. Elementary body composition was measured using a direct segmental multi-frequency bioelectrical impedance analyzer (InBody770; Biospace, Seoul, Korea), and we assessed total fat mass and body fat percentage. This analyzer processes 30 impedance measurements using six different frequencies $(1,5,50,250,500$, and $1,000 \mathrm{kHz})$ at each of five body segments (right arm, left arm, trunk, right leg, and left leg), and 15 reactance measurements using tetrapolar eight-point tactile electrodes using three different frequencies $(5,50$, and $250 \mathrm{kHz})$, at each of the five above-mentioned body segments $[14,15]$. We defined BMFR as body muscle mass divided by the fat mass, and FMR as body fat mass divided by muscle mass $[3,4]$.

\section{Blood sampling and measurement of clinical parameters}

Blood samples for $\mathrm{HbAlc}$, fasting plasma glucose, fasting blood insulin, total cholesterol, low-density lipoprotein (LDL) cholesterol, high-density lipoprotein (HDL) cholesterol, triglycerides, and estimated glomerular filtration ratio (eGFR) were collected from the antecubital vein and analyzed at the Jinnouchi Hospital laboratory. Body weight $(\mathrm{kg})$, height $(\mathrm{cm})$, and waist circumference $(\mathrm{cm})$ were measured in the standing position and body mass index (BMI; $\mathrm{kg} / \mathrm{m}^{2}$ ) was calculated at the same time that body composition was measured.

\section{Statistical analysis}

The Shapiro-Wilk test was used to assess the normal distribution of continuous data. Data were expressed as the mean \pm standard deviation (SD), whereas those with skewed distributions were expressed as the median value with the interquartile range (IQR). We performed simple linear regression analyses, and calculated and compared coefficients of correlation. We evaluated goodness-of-fit using coefficients of determination $\left(\mathrm{R}^{2}\right)$, mean absolute error (MAE) and root mean squared error (RMSE). $\mathrm{P}<0.05$ (two-tailed) was considered significant. Statistical analyses were performed with SPSS ver. 23.0 (IBM Japan, Ltd, Tokyo, Japan).

\section{Results}

\section{Subjects}

We initially enrolled 122 patients with untreated T2DM, but excluded four patients (ketoacidosis: $\mathrm{n}=2$, uncontrolled hypertension: $\mathrm{n}=1$, unable to undergo InBody 770 measurements: $\mathrm{n}=1$ ), for a final cohort of 118 patients. Table 1 shows the participants' characteristics. All subjects had untreated T2DM and 66 patients $(55.9 \%)$ demonstrated high levels of $\mathrm{HbAlc}>$ $8.4 \%(68 \mathrm{mmol} / \mathrm{mol})$, and included 63 patients $(53.3 \%)$ with obesity $\left(\mathrm{BMI} \geq 25 \mathrm{~kg}^{2}\right)$.

\section{Results of body composition analysis}

Analysis of elementary body composition using InBody 770 showed that the total muscle quantity was $45.9 \pm 9.9 \mathrm{~kg}$, total fat quantity was $19.1 \mathrm{~kg}$ (range, $13.2-25.9 \mathrm{~kg}$ ), body fat percentage was $29.0 \pm 9.6 \%$, skeletal muscle mass index (SMI) was 7.47 $\pm 1.23 \mathrm{~kg} / \mathrm{m}^{2}$, BMFR was 2.62 (range, $1.75-3.26$ ), and FMR was 0.38 (range, $0.30-0.54$ ). Seventeen males $(22.7 \%)$ and six females $(14.0 \%)$ were diagnosed with sarcopenia by the Asian Working Group for Sarcopenia (AWGS) criteria [16].

\section{Hyperinsulinemic-euglycemic clamp examination results}

In the total population $(\mathrm{n}=118)$, we measured $\mathrm{M}$ and $\mathrm{M} / \mathrm{I}$ values by hyperinsulinemic-euglycemic clamp examination (M-value: $7.11 \pm 2.89 \mathrm{mg} / \mathrm{m}^{2} / \mathrm{min}, \mathrm{M} / \mathrm{I}$ value: $8.14 \mathrm{mg} / \mathrm{m}^{2} / \mathrm{min}^{2} / \mu \mathrm{IU} \cdot \mathrm{mL}$ (range, $5.02-10.4 \mathrm{mg} / \mathrm{m}^{2} / \mathrm{min}^{2} / \mu \mathrm{IU} \cdot \mathrm{mL}$ )). Number of patients in the insulin resistance $(\mathrm{M} / \mathrm{I}$ value $<9.0)$ was $58(49.2 \%)$.

\section{Simple linear regression analysis}

Body muscle percentage $(\mathrm{B}=0.71, \mathrm{P}<0.01)$ was significantly related to M/I (Fig. 1a); whereas fat percentage $(B=-0.72$, $\mathrm{P}$ 
Table 1. Background Clinical Characteristics of the Study Subjects $(\mathrm{N}=118)$

\begin{tabular}{ll}
\hline Variables & \\
\hline Male $(\%)$ & $55(63.6 \%)$ \\
Age (years) & $56.6 \pm 12.6$ \\
Height $(\mathrm{cm})$ & $163.4 \pm 9.2$ \\
Weight $(\mathrm{kg})$ & $67.0(55.5-76.9)$ \\
Muscle quantity $(\mathrm{kg})$ & $45.9 \pm 9.9$ \\
Body fat quantity $(\mathrm{kg})$ & $19.1(13.2-25.9)$ \\
BMI $\left(\mathrm{kg} / \mathrm{m}^{2}\right)$ & $24.7(21.4-28.6)$ \\
Body fat percentage $(\%)$ & $29.0 \pm 9.6$ \\
Waist circumference $(\mathrm{cm})$ & $90.2 \pm 13.8$ \\
BMFR & $2.62(1.75-3.26)$ \\
FMR & $0.38(0.30-0.54)$ \\
Hemoglobin Alc $(\%)$ & $8.6(7.0-11.0)$ \\
Hemoglobin A1c $(\mathrm{mmol} / \mathrm{mol})$ & $70(52-96)$ \\
Fasting plasma glucose $(\mathrm{mg} / \mathrm{dL})$ & $154(125-209)$ \\
Fasting blood insulin $(\mu \mathrm{U} / \mathrm{mL})$ & $5.4(3.4-7.9)$ \\
HOMA-IR & $2.17(1.22-3.17)$ \\
HOMA- $\beta(\%)$ & $17.3(9.4-30.3)$ \\
Total cholesterol $(\mathrm{mg} / \mathrm{dl})$ & $204(176-228)$ \\
HDL-cholesterol $(\mathrm{mg} / \mathrm{dL})$ & $49(41-57)$ \\
LDL-cholesterol $(\mathrm{mg} / \mathrm{dL})$ & $132.0 \pm 34.2$ \\
Triglycerides $(\mathrm{mg} / \mathrm{dL})$ & $111(71-167)$ \\
eGFR (mL/min/1.73 m²) & $74.9 \pm 17.5$ \\
Current smoking $(\%)$ & $33(28.0)$ \\
Drinking $(\%)$ & $32(27.1)$ \\
\hline & \\
\hline
\end{tabular}

BMI: body mass index; BMFR: body muscle-to-fat ratio; FMR: fat-tomuscle ratio; HOMA-IR: homeostasis model assessment for insulin resistance; HOMA- $\beta$ : homeostasis model assessment for $\beta$ cell function; eGFR: estimated glomerular filtration rate; HDL: high-density lipoprotein; LDL: low-density lipoprotein.

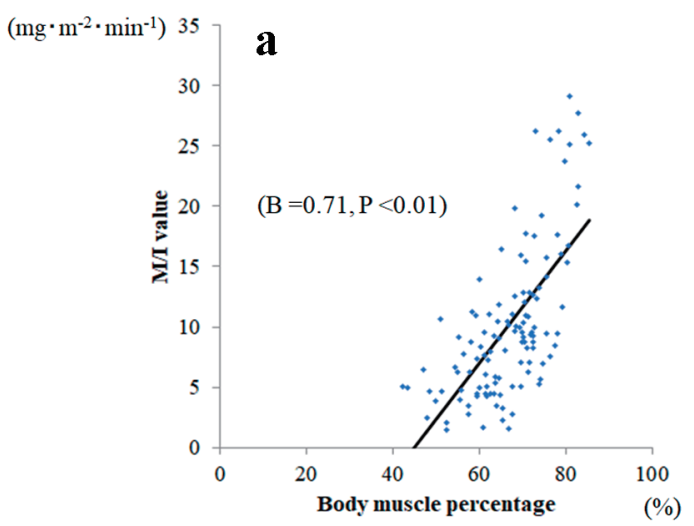

$<0.01$ ) was significantly negatively related to M/I (Fig. 1b). However, the linear fit of body fat percentage to $\mathrm{M} / \mathrm{I}$ ratio was not good. The reciprocal approximation curve of body fat percentage (red, $\mathrm{R}^{2}=0.61$ ) fits was better than the approximate linear line of body fat percentage (black, $\mathrm{R}^{2}=0.43$ ).

Simple linear regression analyses confirmed correlations between M/I and BMFR (B: $0.756(\mathrm{P}<0.01), \mathrm{R}^{2}$ : 0.572, MAE: 3.19, and RMSE: 4.14), and between M/I and FMR (B: -0.601 $(\mathrm{P}<0.01), \mathrm{R}^{2}$ : 0.362, MAE: 3.97, and RMSE: 5.05; Fig. 2). Against the M/I values, BMFR also showed better goodnessof-fit than did FMR. In comparing correlation coefficients, the BMFR absolute $B$ value was significantly larger than that of FMR $(\mathrm{P}=0.027)$.

To correct BMFR and FMR by weight to avoid the effects of dehydration, a simple regression sub-analysis of BMFR per body weight and FMR per body weight M/I values was performed. The correlation with $\mathrm{M} / \mathrm{I}$ values was stronger for BMFR per weight $(\mathrm{B}=0.78, \mathrm{P}<0.01)$ than for $\mathrm{FMR}$ per weight $(\mathrm{B}=0.42, \mathrm{P}<0.01)$.

\section{Discussion}

The present study shows that the correlation between BMFR (as a combined body composition measurement index) and $\mathrm{M} / \mathrm{I}$ (which is a definitive index of IR) is significantly stronger and closer than that between FMR and M/I.

Recent studies have suggested the potential usefulness in managing metabolic diseases of an index that combines muscle mass and fat mass $[17,18]$. Previously, we reported that BMFR has a strong linear correlation with $\mathrm{M} / \mathrm{I}[1,2]$. It is reported that T2DM is associated with increased risks of sarcopenia and pre-sarcopenia [19], and this study also included 23 patients $(19.5 \%)$ with sarcopenia. Further, $53.3 \%$ of the participants in this study were obese (BMI $\geq 25 \mathrm{~kg} / \mathrm{m}^{2}$ ), and body muscle percentage is biased towards a lower range compared to healthy subjects. Therefore, BMFR, which has a wide range of numerical values, may be more suitable for quantitative evaluation of IR than FMR in T2DM patients. Other, in the relationship between the fat percentage and $\mathrm{M} / \mathrm{I}$, we first found

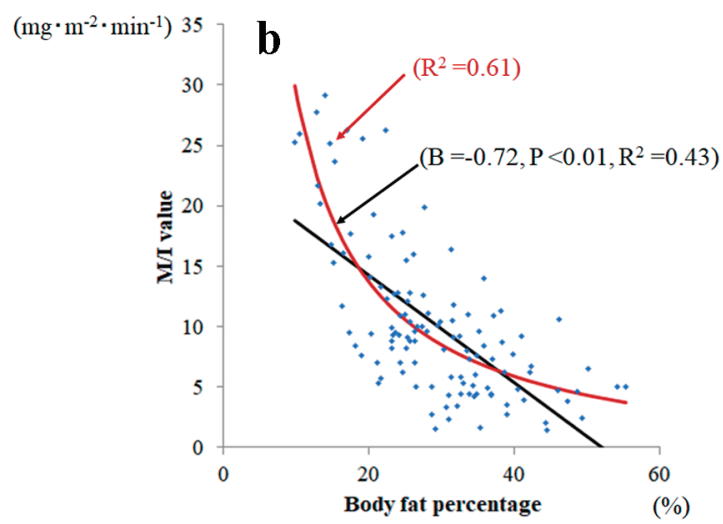

Figure 1. Relationship between body muscle percentage and body fat percentage and $\mathrm{M} / \mathrm{I}$. (a) Body muscle percentage was significantly related to $M / I$ value $(B=0.71, P<0.001)$, whereas $(b)$ body fat percentage was significantly and negatively related to $M / I(B=-0.72, P<0.001)$. Among body fat percentages, the reciprocal approximation curve (red line; $\left.R^{2}=0.61\right)$ fits better than the approximate line (black line; $R^{2}=0.43$ ) against $M / I$ values. 

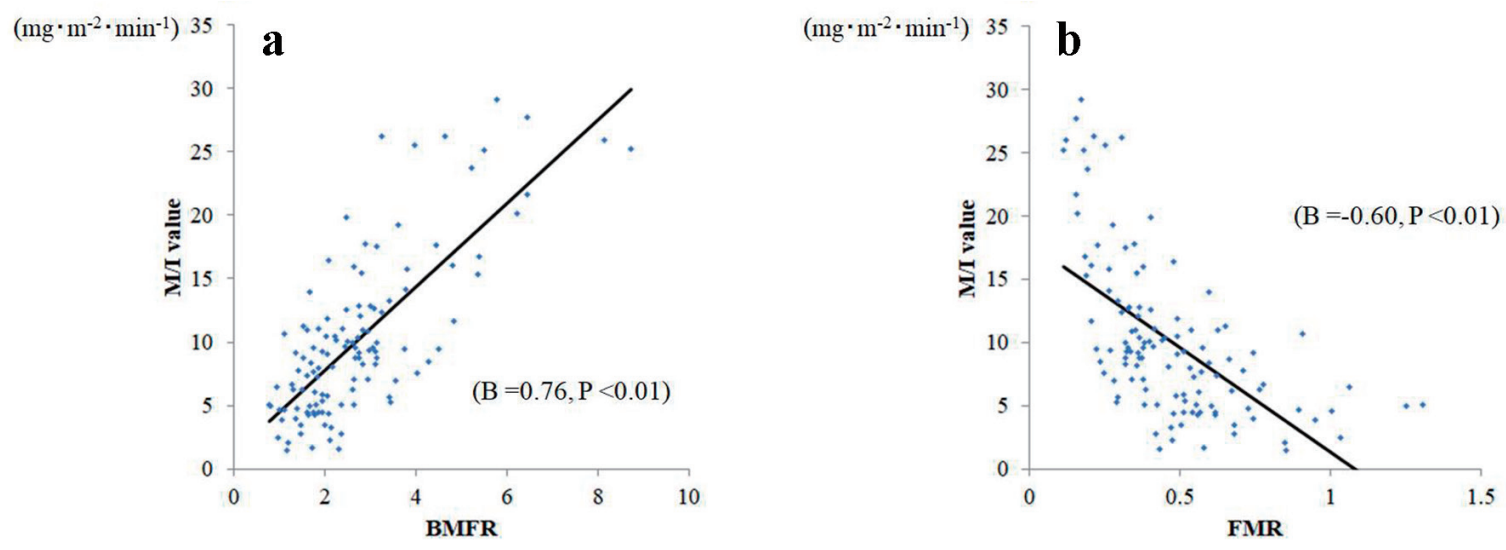

Figure 2. Relationship between body muscle-to-fat ratio (BMFR) and fat-to-muscle ratio (FMR) and M/I. (a) BMFR was significantly related to $M / I(B=0.76, P<0.001)$, whereas $(b) F M R$ was significantly and negatively related to $M / I(B=-0.60, P<0.001)$.

that goodness-of-fit for the reciprocal approximation curve of fat percentage was better than the approximate line of fat percentage. The reciprocal values of fat percentage and muscle per body weight were positively correlated with M/I. Using these two positively correlated parameters, BMFR, which was obtained by multiplying the reciprocal values of fat percentage by muscle percentage $(\mathrm{BMFR}=$ (body muscle percentage: muscle mass $\times 100 /$ body weight $) \times($ reciprocal values of body fat percentage: body weight/fat mass $\times 100)$ ) had a stronger linear regression correlation with M/I than did FMR.

This study has limitations. Subjects were relatively few, and were confined to Japanese patients with untreated T2DM. Nonetheless, simple assessment of IR using BMFR in healthy individuals may be a good indicator of early intervention in T2DM prevention. Consequently, further studies are warranted to verify our results in a population that includes healthy people.

In conclusion, BMFR is more useful than FMR in quantifying IR in patients with T2DM because the correlation between BMFR and the insulin sensitivity index M/I is significantly greater than that between FMR and M/I.

\section{Acknowledgments}

None to declare.

\section{Financial Disclosure}

None to declare.

\section{Conflict of Interest}

HJ has received honoraria from Novo Nordisk, Sanofi, AstraZeneca Pharmaceuticals, Astellas Pharma, Boehringer Ingelheim, Daiichi-Sankyo, Eli Lilly, Takeda, and Novartis Pharmaceuticals. SS has received honoraria from MSD, AstraZeneca Pharmaceuticals, Ono Pharmaceutical, Bayer Yakuhin, Ltd, and Novo Nordisk. There is no other potential conflict of interest relevant to this article.

\section{Informed Consent}

Signed informed consent was obtained from each patient.

\section{Author Contributions}

NK, SS, MN and HJ contributed to the analysis design, acquisition and interpretation of data and reviewed/edited the manuscript. AY, KH, FM, KK, KJ and TJ contributed to the interpretation of data and reviewed/edited the manuscript. All authors read and approved the final manuscript.

\section{Data Availability}

The authors declare that data supporting the findings of this study are available within the article.

\section{References}

1. Kurinami N, Sugiyama S, Yoshida A, Hieshima K, Miyamoto F, Kajiwara K, Jinnouchi T, et al. Correlation of body muscle/fat ratio with insulin sensitivity using hyperinsulinemic-euglycemic clamp in treatment-naive type 2 diabetes mellitus. Diabetes Res Clin Pract. 2016;120:65-72.

2. Kurinami N, Sugiyama S, Yoshida A, Hieshima K, Miyamoto F, Kajiwara K, Jinnouch K, et al. Body muscleto-fat ratio gender-specific cut-off values for impaired insulin sensitivity in patients with treatment-naive type 2 diabetes mellitus. Endocrine. 2019;66(3):503-508.

3. Xu K, Zhu HJ, Chen S, Chen L, Wang X, Zhang LY, Pan $\mathrm{L}$, et al. Fat-to-muscle ratio: a new anthropometric indicator for predicting metabolic syndrome in the Han and Bouyei populations from Guizhou province, China. Bi- 
omed Environ Sci. 2018;31(4):261-271.

4. Ramirez-Velez R, Carrillo HA, Correa-Bautista JE, Schmidt-RioValle J, Gonzalez-Jimenez E, Correa-Rodriguez M, Gonzalez-Ruiz K, et al. Fat-to-Muscle Ratio: A New Anthropometric Indicator as a Screening Tool for Metabolic Syndrome in Young Colombian People. Nutrients. 2018;10(8):1027.

5. Giannarelli R, Aragona M, Coppelli A, Del Prato S. Reducing insulin resistance with metformin: the evidence today. Diabetes Metab. 2003;29(4 Pt 2):6S28-35.

6. Miyazaki Y, Mahankali A, Wajcberg E, Bajaj M, Mandarino LJ, DeFronzo RA. Effect of pioglitazone on circulating adipocytokine levels and insulin sensitivity in type 2 diabetic patients. J Clin Endocrinol Metab. 2004;89(9):4312-4319.

7. Merovci A, Solis-Herrera C, Daniele G, Eldor R, Fiorentino TV, Tripathy D, Xiong J, et al. Dapagliflozin improves muscle insulin sensitivity but enhances endogenous glucose production. J Clin Invest. 2014;124(2):509-514.

8. Yki-Jarvinen H, Koivisto VA. Insulin sensitivity in newly diagnosed type 1 diabetics after ketoacidosis and after three months of insulin therapy. J Clin Endocrinol Metab. 1984;59(3):371-378.

9. DeFronzo RA, Tobin JD, Andres R. Glucose clamp technique: a method for quantifying insulin secretion and resistance. Am J Physiol. 1979;237(3):E214-223.

10. Kurinami N, Sugiyama S, Morita A, Yoshida A, Hieshima K, Miyamoto F, Kajiwara K, et al. Ratio of muscle mass to fat mass assessed by bioelectrical impedance analysis is significantly correlated with liver fat accumulation in patients with type 2 diabetes mellitus. Diabetes Res Clin Pract. 2018;139:122-130.

11. Kurinami N, Sugiyama S, Ijima H, Yoshida A, Hieshima K, Miyamoto F, Kajiwara K, et al. Clinical usefulness of the body muscle-to-fat ratio for screening obstructive sleep apnea syndrome in patients with inadequately controlled type 2 diabetes mellitus. Diabetes Res Clin Pract.
2018;143:134-139.

12. Kurinami N, Sugiyama S, Nishimura H, Morita A, Yoshida A, Hieshima K, Miyamoto F, et al. Clinical factors associated with initial decrease in body-fat percentage induced by add-on sodium-glucose co-transporter 2 inhibitors in patient with type 2 diabetes mellitus. Clin Drug Investig. 2018;38(1):19-27.

13. Kurinami N, Sugiyama S, Yoshida A, Hieshima K, Miyamoto F, Kajiwara K, Jinnouch K, et al. Dapagliflozin significantly reduced liver fat accumulation associated with a decrease in abdominal subcutaneous fat in patients with inadequately controlled type 2 diabetes mellitus. Diabetes Res Clin Pract. 2018;142:254-263.

14. Cha K, Chertow GM, Gonzalez J, Lazarus JM, Wilmore DW. Multifrequency bioelectrical impedance estimates the distribution of body water. J Appl Physiol (1985). 1995;79(4):1316-1319.

15. Cha K, Shin S, Shon C, Choi S, Wilmore DW. Evaluation of segmental bioelectrical impedance analysis (SIBA) for measuring muscle distribution. J ICHPER SD-ASIA. 1997:11-14.

16. Chen LK, Liu LK, Woo J, Assantachai P, Auyeung TW, Bahyah KS, Chou MY, et al. Sarcopenia in Asia: consensus report of the Asian Working Group for Sarcopenia. J Am Med Dir Assoc. 2014;15(2):95-101.

17. Seo YG, Song HJ, Song YR. Fat-to-muscle ratio as a predictor of insulin resistance and metabolic syndrome in Korean adults. J Cachexia Sarcopenia Muscle. 2020;11(3):710-725.

18. Chen YY, Fang WH, Wang CC, Kao TW, Yang HF, Wu CJ, Sun YS, et al. Fat-to-muscle ratio is a useful index for cardiometabolic risks: A population-based observational study. PLoS One. 2019;14(4):e0214994.

19. Wang T, Feng X, Zhou J, Gong H, Xia S, Wei Q, Hu X, et al. Type 2 diabetes mellitus is associated with increased risks of sarcopenia and pre-sarcopenia in Chinese elderly. Sci Rep. 2016;6:38937. 